\title{
Determination of Relative Frequency of Calretinin Expression in Patients with Breast Cancer in Yazd,
}

\section{Iran}

\author{
Ehsan Arjmandzadeh ${ }^{1}$, Fariba Binesh ${ }^{2,3}$ \\ 1 Shahid Sadoughi University of Medical Sciences, International branch, Yazd, Iran \\ 2 Department of Pathology, Shahid Sadoughi University of Medical Sciences, Yazd, Iran \\ 3 Hematology and Oncology Research Center, Shahid Sadoughi University of Medical Sciences and Health Services, Yazd, Iran \\ *Corresponding Author: Dr. Fariba Binesh, 1 Department of Pathology, Shahid Sadoughi University of Medical Sciences, Yazd, \\ Iran. 2 Hematology and Oncology Research Center, Shahid Sadoughi University of Medical Sciences and Health Services, Yazd, \\ Iran. \\ Email: binesh44@yahoo.com; Tel: 983518113698
}

Received: April 13, 2020; Accepted: June 22, 2020

\begin{abstract}
Introduction: Breast cancer is known as the most common type of cancer among women in the world. Several methods have been proposed to predict the behavior of breast carcinoma. Recently calretinin has been found to be a reliable factor in predicting tumor survival rate in breast cancer. The aim of this study was to evaluate the relative frequency of calretinin expression in patients with breast cancer in Yazd,Iran. Material and methods: In this cross-sectional retrospective study, the clinicopathologic features and the outcome of patients with breast cancer from 2017 to 2018 were reviewed at Shahid Sadoughi Hospital, Yazd, Iran. The influence of potential prognostic parameters in the overall survival was investigated by log-rank test and Cox regression analysis. Results: Among 100 cases with breast carcinoma, 14.5\% were positive for calretinin. The distribution of the rate of positivity of IHC markers including ER, PR, P53, Ki67 and Her2 was $62.9 \%, 57.1 \%, 46.2 \%, 80.5 \%$ and $22.1 \%$, respectively. The most common grade and stage were grade2 and stage3 respectively. There was no significant difference in the status of IHC markers (including ER, PR, Her2 and Ki67) in terms of calretinin. $66.7 \%$ of the patients with calretinin positive results were P53 positive. The results of the mean tumor size distribution in the two groups of positive and negative calretinin showed a significant difference $(P$-value $=0.05)$. A lower age at the time of diagnosis was found in patients with calretinin positive results $(P$-value $=0.119)$. The mean survival rate in calretinin positive group was 6.71 years and 6.62 years in calretinin negative patients which was not statistically significant. Conclusion: The results of this study indicated an association between calretinin expression and other IHC markers (although not statistically significant) in predicting poor prognosis in breast cancer patients. In addition, we found a statistically significant association among calretinin with smaller tumor size and lower age at the time of diagnosis in patients with breast cancer.
\end{abstract}

Keywords: Breast cancer, Calretinin. Survival.

\section{INTRODUCTION}

Cancer is one of the most important human health challenges today. It is currently the second leading cause of death in developed countries and the third most common cause of death in developing countries [1]. Meanwhile, breast cancer is also known as the most common type of cancer among women in the world (about $27 \%$ of all cancers), and the first-ever death (about $16 \%$ of total mortality) is due to breast cancer in women [2]. According to recent studies, among Iranian women, breast cancer account for $24.4 \%$ of the total cancers, and out of a total of 6160 new cases of breast cancer diagnosed in a year. It is claimed that breast cancer kills 40,000 Iranian women a year, and yearly 70,000 new cases of breast cancer are also recorded [1]. Based on review articles, the incidence of breast cancer in Iran is about 10 years lower than that of developed countries [1, 3]. To date, several methods have been proposed to predict the behavior of breast carcinoma and even the classification derived from the genomic analysis of this malignant tumor is available. But it currently does not have the clinical efficacy and is limited 
to researches. Therefore, achieving this goal is still mainly based on the clinical-pathological characteristics of the tumors, including size, tumor grade, lymph node involvement, the status of hormonal receptors, and Human Epidermal Growth Factor receptor 2 (HER-2). In spite of all the progress made, in many cases the behavior of breast tumors is unpredictable [4]. Recently calretinin, previously thought to be found exclusively in neurons and mesothelial cells, has been found to be a reliable factor in predicting tumor grade, prognosis and survival rate of breast cancer patients $[4,5,6,7]$. Calretinin is a vitamin Ddependent intracellular protein, belonging to calcium-binding calmodulin family, and plays a role in regulating intracellular calcium level and transmitting messages $[5,6]$. Considering the high prevalence of breast cancer and the lower age incidence rates in Iran relative to the global average, we intended to study the relative frequency of calretinin expression in patients with breast cancer in Yazd, Iran.

\section{MATERIAL AND METHODS}

In this cross sectional, retrospective project, the medical records of the patients from 2017 to 2018 were reviewed at Shahid Sadoughi Hospital Yazd, Iran. Sampling was done by taking a census. The medical charts of all patients diagnosed with breast carcinoma were retrieved. As study variables, we recorded the patient's hospital registration number; date of admission; and personal information, including age, the side of the breast involved, type of tumor, grade, stage, IHC results including ER, PR, HER2, P53, Ki67, calretinin and survival. Data were obtained via the patients' records and by phone. Overall survival (OS) was calculated from date of diagnosis until death or the date of the last follow-up. The patients were staged according to the tumor, node, and metastasis (TNM) staging system. Survival data were analyzed using Kaplan-Meier estimates, and multivariate analysis was performed using the Cox regression method. A p-value $<0.05$ was considered statistically significant. Statistical analyses were performed using IBMC SPSS(C) Statistics,version 22 (IBMC Corp., Armonk, NY, USA). Primary (calretinin) and secondary (HRP) antibodies necessary for IHC were prepared from Tehran and their characteristics are as follow: calretine from Dako (USA) with code IS627-D-1 (as an initial antibody) and HRP from Zytochem (Germany) with ZUC053-006 code.

This work was conducted in accordance with the Declaration of Helsinki (2000). This study was approved by the Ethics Committee of Shahid Sadoughi University of Medical Sciences, Yazd,Iran , after which the forms for recording demographic data were completed.

\section{RESULTS}

At first there were 176 cases; however, due to lack of some tissue blocks necessary for IHC method, this figure reduced to 100 cases. Among 100 cases with breast carcinoma $14.5 \%$ were positive for calretinin. Generally, the most common type of breast cancer was infiltrating ductal carcinoma (IDC) $(87.7 \%)$ followed by infiltrating lobular carcinoma (ILC) $(9.6 \%)$ and medullary carcinoma (MC) $(2.6 \%)$. The right breast was involved in about $54.1 \%$. The distribution of the rate of positivity of $\mathrm{IHC}$ markers including ER, PR, P53, Ki67 and Her2 was $62.9 \%$, $57.1 \%, 46.2 \%, 80.5 \%$ and $22.1 \%$, respectively. By assessing the grade of breast cancer cases, the most common grade was grade 2 with a frequency of $39.3 \%$. Grade 3 and 1 were with the frequency of $38.3 \%$ and 22.4 respectively. Regarding stage of the tumor, the most commonly reported stage was stage-3 with $44.4 \%$. There was no statistically significant difference between the two groups with calretinin positive or negative results in relation to the involved side. There was no significant difference in the status of IHC markers (including ER, PR, Her2 and Ki67) in terms of calretinin. ER and PR were negative in $55.6 \%$ of the patients with positive calretinin results and the majority of calretinin negative cases were ER and PR negative $(66.7 \%$ and $61.4 \%$ respectively). $66.7 \%$ of the patients with calretinin positive result were P53 positive. The majority of Her2 negative cases $(66.7 \%$ ) were calretinin positive. In this study, all calretinin positive patients were positive for Ki67, but only $9.1 \%$ of negative calretinin cases were associated with negative Ki67. The results of the mean tumor size distribution in the two groups of positive and negative calretinin showed a significant difference $(P$-value $=0.05)$, in which the mean tumor size in the calretinin negative group was $5.3 \mathrm{~cm}$ and the mean tumor size of calretinin positive group was 4.0- $\mathrm{cm}$ (table-1). The mean age of the patients in the two groups of positive and negative calretinin was not statistically significant $(P$-value $=0.119)$, but a lower age at the time of diagnosis was found in patients with calretinin positive results (44.25 vs 49.7 ys). Overall survival regardless of the association with other clinical and pathological indicators was 6.81 years (with a range of 5.95 to 7.67) (fig-1). The mean survival time according to the grade of the tumor also showed that there was the lowest survival in grade 2 ( 5.68 years) and the highest survival rate in grade 1 (7.78 years). The mean survival rate in calretinin positive group was 6.71 years and 6.62 years in calretinin negative patients which was not statistically significant (table-2, fig-2).

\section{DISCUSSION}

In this study, we compared the clinical and pathological parameters as well as survival time in two groups of positive and negative calretinin in a total of 100 patients with breast cancer. A few similar studies have been conducted in the world, but no similar study was found in Iran. In this study, about $14.5 \%$ of the patients with breast cancer were positive for calretinin. While other studies have reported between 21 and $32 \%(4,5,6,7,8)$. The relative frequency of the tumor type was $87.7 \%$ for IDC type (88\% in similar studies), $9.6 \%$ for ILC type $(7.6 \%$ in similar studies) and $2.6 \%$ for the MC type (4.4\% in similar studies), which is consistent with other works [7]. Our results revealed that grade 2 and stage 3 were the most common grade and stage. It was consistent with previous studies [4,7]. Similar to other studies the present study showed that ER and PR positive specimens were more frequent than hormone receptor negative samples [4, 7, 9]. $70.6 \%$ of our patients were Her2 negative which is in contrast with others3.4. To explain this difference, we should say that this may be due to the different type of kit or antibody used, diverse bounds applied to define the test result, or the different interpretation of the IHC test results by pathologists.

$19.5 \%$ of the cases were negative for Ki67 and $80.5 \%$ were positive that is similar to other works $[10,11,12]$. P53 were negative in $53.8 \%$ of the patients and positive in $46.2 \%$. Our negative results were more common than similar projects $[7,13]$. In this research, there was no association between calretinin results and the side of breast involvement. This topic is not investigated in other studies. In the present study, the mean age of the patient with breast cancer at the time of diagnosis was significantly different between the calretinin positive and negative groups. Despite the lack of a strong statistical relationship, it suggests a clear tendency for more frequent calretinin expression in younger patients. Studies that were done in Iran and other eastern countries did not find a meaningful relationship between age at the time of diagnosis and prognosis of breast cancer patients but Western studies 
have shown that lower age at the time of diagnosis is with a worse prognosis, more tumor invasiveness, more probability of negative hormonal receptors and the Her2 expression(triple negative). Therefore, in this context, our study results are closer to the western ones $[14,15,11]$. Our results revealed that $55.6 \%$ of calretinin positive patients lack ER hormone receptor although it was not statistically significant. This figure was $87.5 \%$ in other studies $[7,1]$. The positive ER rate in calretinin negative samples was $66.7 \%$ in our estimates and $86 \%$ in other studies $[7,1]$. As a result, it seems that calretinin positive breast cancer is more likely to be ER negative [7,1]. It is true about PR, too [4, 7].66.7\% of calretinin positive specimen were Her2 negative and $44.6 \%$ of calretinin negative samples were Her2 positive. This was in contrast with other studies [7]. To explain this difference, we should say that although there is a clear guideline regarding the expression of Her2 results, there is disagreement between pathologists about interpretation of score 2 and3. All calretininpositive patients were Ki67-positive (48.6\% in similar studies) but only $9.1 \%$ of calretinin-negative cases were Ki67-negative (75.5\% in similar studies). $66.7 \%$ of calretinin-positive patients were found to be P53-positive, and $55.6 \%$ of P53 negative were in calretinin-negative samples $[8,16,10,13]$. A similar study comparing P53 with calretinin expression was not found. Our study revealed that breast tumor size was smaller in calretinin positive patients, it was statistically meaningful. There was no similar study in the literature. In this study, overall survival in patients with breast cancer regardless of age, IHC markers, and tumor grade and stage were about 6.81 years. 5-year survival rate was $55 \%$. This figure in other researches is $61.2 \%[1,14,15]$. Regarding grade and overall survival there was a significant relationship between grade and overall survival, the highest survival rate of 7.78 years was seen in grade 1 cases, $(p=0.01)$ Calretinin negative patients survived 6.62 years $(4.41$ years in similar studies) and calretinin positive patients 6.71 years. (3.75 years in similar studies). Statitical analysis showed that the mean survival rate of patients with positive and negative calretinin results was not statistically significant $(p=0.388)$. However, the majority of other studies have emphasized on the significant association between calretinin positivity and lower survival rate. The probable reasons for this dissimilarity could be related to:1-The inadequate frequency of positive calretinin samples in the present work compared to other studies $(14 \%$ vs. $30 \%$ ), although the duration of follow-up and overall sample size were comparable 2- Since calretinin positivity usually exists in association with other negative prognosis predictors such as triple negativity, these patients received more aggressive treatment protocols $[4,5]$.

In other studies, the relationship between calretinin and survival according to grade were emphasized. They showed that there was a strong association between calretinin positivity and the shortest survival in grade 3 tumors $[4,5,8,16]$. We also found similar results, although it was not statistically significant $(p=0.757)$. The results of this study indicated an association between calretinin with other IHC markers (although not statistically significant) in predicting poor prognosis in breast cancer patients. In addition, we found a statistically significant association among calretinin with smaller tumor size and lower age at the time of diagnosis in patients with breast cancer.

It seems that in breast cancer, calretinin will be able to play a role in predicting survival and oncotyping along with other common markers in the future.
Table 1: Comparison of mean tumor size based on calertinin expression in patients with breast carcinoma

\begin{tabular}{cc}
\hline Calretinin & Mean tumor size \pm standard deviation \\
\hline Negative & $5.30 \pm 4.15$ \\
Positive & $4.00 \pm 1.86$ \\
\hline
\end{tabular}

Table 2: Comparison of mean survival based on calretinin expression status in patients with breast carcinoma

\begin{tabular}{cc}
\hline Calretinin & Mean survival (years) \pm standard error \\
\hline Negative & $6.62 \pm 0.45(5.72-7.51)$ \\
Positive & $6.71 \pm 0.74(5.26-8.17)$ \\
Total & $6.79 \pm 0.43(5.93-7.65)$ \\
\hline
\end{tabular}

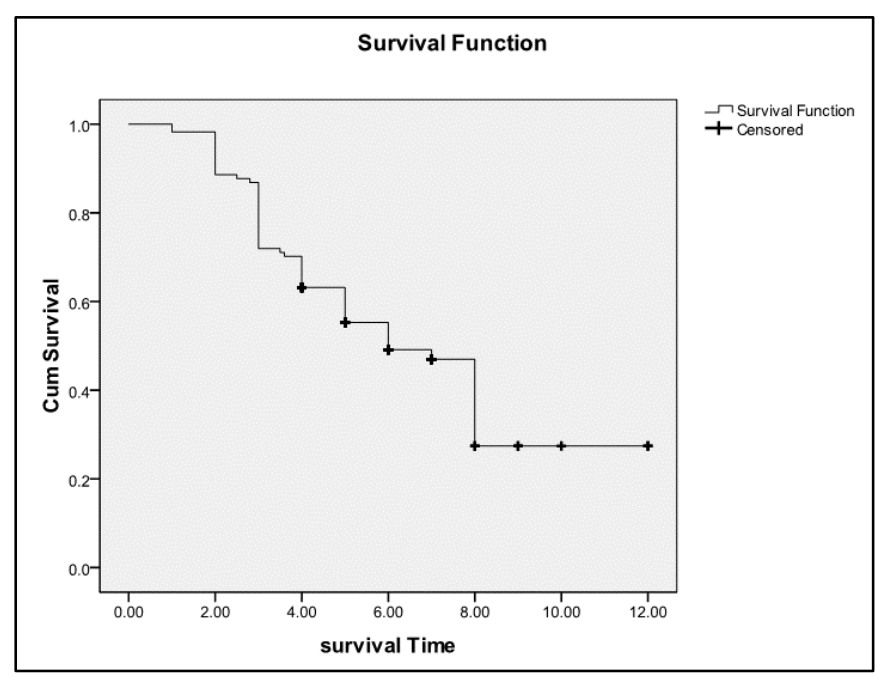

Fig 1: Overall survival of patients with breast cancer

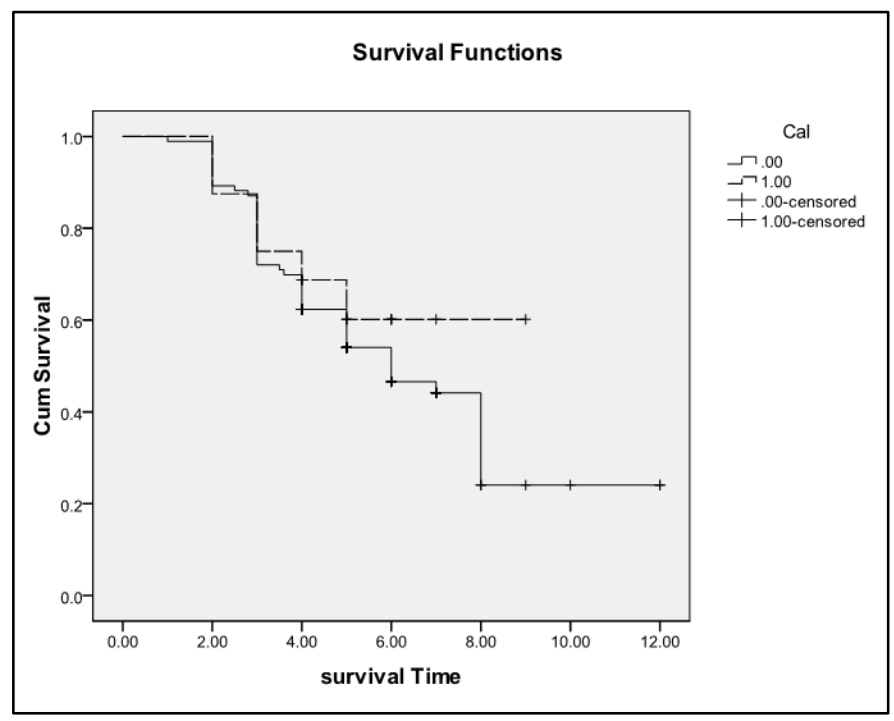

Fig 2: Comparison of survival of patients with breast cancer in two groups of positive and negative calretinin

\section{Acknowledgements}

None.

Contributions: All the authors contributed in the preparation of this research and article. 


\section{REFERENCES}

1. Mahdavifar $\mathrm{N}$ et al. "Spatial Analysis of Breast Cancer Incidence in Iran.", Asian Pac J Cancer Prev, 2016;17(S3): 59-64

2. Hosseinzadeh $\mathrm{M}$ et al. "Risk factors for breast cancer in Iranian women: a hospital-based case-control study in tabriz, iran." J Breast Cancer, 2014;17(3):236-243.

3. Rahimzadeh $\mathrm{M}$ et al. "Survival Rates for Breast Cancer in Iranian Patients: a Meta- Analysis." Asian Pacific Journal of Cancer Prevention,2016;17(4):2223-2227.

4. Taliano RJ et al. "Calretinin expression in high-grade invasive ductal carcinoma of the breast is associated with basal-like subtype and unfavorable prognosis." Hum Pathol, 2013;44(12):2743-2750.

5. Farrag MS, et al. "Calretinin expression as a reliable prognostic marker in different molecular subtypes of breast carcinoma." Indian J Pathol Microbiol, 2017; 60(1):8-14.

6. Powell G, et al. "Expression of calretinin by breast carcinoma and the potential for misdiagnosis of mesothelioma." Histopathology. 2011;59(5):950-956.

7. Mayada S, et al. "Clinicopathological correlation of calretinin expression in breast cancer". Zum J. 2016;22(4):181-189

8. Lugli A et al. "Calretinin expression in human normal and neoplastic tissues: a tissue microarray analysis on 5233 tissue samples." Hum Pathol, 2003;34(10):994-1000

9. Elston CW, Ellis IO. "Pathological prognostic factors in breast cancer. I. The value of histological grade in breast cancer: experience from a large study with long-term followup. C. W. Elston \& I. O. Ellis. Histopathology 1991; 19; 403410." Histopathology, 2002;41(3A):151-152, discussion 152-153.

10. Dowsett $\mathrm{M}$, et al. "Assessment of $\mathrm{Ki} 67$ in breast cancer: recommendations from the International Ki67 in Breast Cancer working group." J Natl Cancer Inst, 2011;103(22):1656-1664.

11. Kao SC, et al. "Low calretinin expression and high neutrophil-to-lymphocyte ratio are poor prognostic factors in patients with malignant mesothelioma undergoing extrapleural pneumonectomy." J Thorac Oncol, 2011;6(11):1923-1929.

12. Altaf FJ, et al. "Metaplastic carcinoma of the breast: an immunohistochemical study." Diagn Pathol. 2014;9:139.

13. Elkablawy MA, et al. "Ki67 expression in breast cancer. Correlation with prognostic markers and clinicopathological parameters in Saudi patients." Saudi Med J, 2016;37(2):137-141

14. Vostakolaei FA, et al. "Age at diagnosis and breast cancer survival in iran." Int J Breast Cancer, 2012, 517976.

15. Brenner DR, et al. "Breast cancer survival among young women: a review of the role of modifiable lifestyle factors." Cancer Causes Control,2016;27(4):459-472

16. Schwaller B. "Calretinin: from a "simple" $\mathrm{Ca}\left(2^{+}\right)$buffer to a multifunctional protein implicated in many biological processes." Front Neuroanat, 2014;8:3 